\title{
Protocolo SUS como ferramenta de verificação do nível de satisfação na interação com tipos com serifa e sem serifa aplicados em mídias digitais e impressas em atividade de leitura. \\ Satisfaction level in the interaction with serif and sans serif typefaces presented in digital and printed media
}

\author{
João Marcelo Ribeiro Soares
}

usabilidade, satisfação, tipografia, sus (system usability scale)

\begin{abstract}
O artigo apresenta um estudo com o objetivo de contribuir com uma metodologia para verificar o nível de satisfação da tipografia após uma tarefa de leitura. No estudo foram utilizados dois tipos de letra, sendo uma com serifa (Garamond) e outra sem (Franklin Gothic). Os leitores realizaram a tarefa de leitura em mídia impressa e digital. O estudo investigou um total de 84 estudantes com idade entre 18 e 24 anos. O protocolo de pesquisa utilizado foi uma adaptação do System Usability Scale (SUS). Na composição foram controladas variáveis como: texto, número de caracteres por linha, entrelinha e altura-x. Nesta última variável o tamanho do corpo foi ajustado por meio de aproximação ótica. Os resultados não apresentaram diferença significativa $(p>0,05)$ entre os tipos de letras e as mídias utilizadas.
\end{abstract}

usability, satisfaction, typeface, sus (system usability scale)

This study proposes to verify the level of satisfaction of readers of continuous texts, during the interaction with different typefaces: Garamond and Franklin Gothic (with and without serifs) and reading different media (digital and printed). Participants in the study were 84 students aged between 18 and 24 years old. Two reading devices were used (a tablet and prototypes) and a System Usability Scale (SUS) protocol was applied. The $x$-height of typefaces was a determining factor in the control of the variables. The results showed that there was no significant difference $(p>0.05)$ in relation to the different typefaces used, and to the different media.

\section{Introdução}

A atividade de leitura é um sistema que envolve um sujeito, uma motivação, uma condição ambiental e um objeto que materializa o texto e possibilita a interação.

Atualmente o texto pode ser materializado por meio de uma publicação convencional impresso em papel (revista, livro, jornal, etc.) ou na forma digital (tablet, celular ou computador). Independente do objeto de leitura utilizado, um componente essencial à realização da atividade de leitura é a tipografia, fato que motiva pesquisadores e profissionais na busca de instrumentos e técnicas que permitam identificar e validar aspectos que tornem os textos mais eficientes à leitura.

As pesquisas sobre legibilidade tipográfica datam desde 1790 (Geske, 1996), e as justificativas para realização de tais pesquisas são muitas, fatores como reduzir a fadiga visual, combater a proliferação da miopia, aumentar a velocidade de captação de informação escrita, facilitar a compreensão, tornar o processo de leitura mais confortável. (Lund, 1999).

Em 1999, Ole Lund fez uma revisão em 30 pesquisas que investigavam a influência do

Anais do 9 $\mathrm{CIDI}$ e 9 CONGIC

Luciane Maria Fadel, Carla Spinillo, Anderson Horta, Cristina Portugal (orgs.)

Sociedade Brasileira de Design da Informação - SBDI

Belo Horizonte | Brasil | 2019

ISBN 978-85-212-1728-2
Proceedings of the 9th CIDI and 9th CONGIC

Luciane Maria Fadel, Carla Spinillo, Anderson Horta, Cristina Portugal (orgs.)

Sociedade Brasileira de Design da Informação - SBDI

Belo Horizonte | Brazil | 2019

ISBN 978-85-212-1728-2 
Soares, J. M. R. | Protocolo SUS como ferramenta de verificação do nível de satisfação na interação com tipos com serifa e sem serifa aplicados em mídias digitais e impressas em atividade de leitura.

tipo de letra no processo de leitura, publicadas entre os anos de 1896 e 1997, como resultado o autor aponta vários problemas em relação aos processos metodológicos utilizados, além disso aponta a fragilidade dos resultados apresentados em tais pesquisas.

Desde então, novos esforços foram realizados neste contexto buscando respostas às questões sobre as influências dos tipos de letras no processo de leitura, muitos contando com a participação de especialistas em tipografia para um melhor preparo das amostras a serem analisadas e também buscando adaptar novas metodologias e tecnologias, a fim de controlar com um pouco mais de precisão as variáveis envolvidas na tarefa de leitura.

Entre os estudos mais recentes sobre os efeitos da aparência das letras no processo de leitura, Beier, Sand e Starrfelt (2017) fortalecem o conceito de que a eficiência máxima da legibilidade está em respeitar o esqueleto da letra, garantindo a identificação do signo que o leitor está acostumado ou familiaridade tipográfica (Beier, 2009).

Resultado também encontrado nos estudos de Soares (2016) que concluiu que fatores simbólicos e estéticos adicionados ao esqueleto das letras, sem alterar seus padrões de maneira significativa, são irrelevantes para o processo de leitura.

Soleimani \& Mohammadi (2012) também não encontraram efeitos relevantes na tarefa de leitura comparando letras diferentes, com e sem serifa. Resultados semelhantes obtiveram Arditi \& Cho (2005) sobre a influência das serifas no processo de leitura.

$O$ fato de o leitor ignorar a aparência das letras para focar no esqueleto delas tem uma função prática, uma vez que tal procedimento busca favorecer a comunicação, recurso chamado de "invariância perceptiva", que é a capacidade que os neurônios têm de perceber detalhes e ao mesmo tempo ignora-los por uma questão de prioridades.

Operacionalmente, para que o processo de leitura ocorra de maneira mais eficiente ignora-se detalhes como a aparência física para que a atenção esteja no cerne da tarefa, no caso, identificar os signos e seus padrões para decodificar a mensagem. Quer dizer, a forma da letra é irrelevante, se respeita as convenções vigentes da linguagem escrita.

Com base na conjuntura apresentada, este estudo busca explorar a adaptação do protocolo System Usability Scale (SUS) na verificação da influência da letra no processo de leitura.

Nesse momento duas questões podem surgir: Por que fazer um estudo para verificar algo que já foi esclarecido? E por que foi escolhida tal ferramenta?

Explico, se a questão central que é se a aparência da letra influência no processo de leitura já foi respondida, a resposta pode ser utilizada como parâmetro para validar novas metodologias de verificação, neste caso buscando procedimento e ferramenta mais simples, que permitam ampliar os conhecimentos sobre tipografia. A justificativa para a escolha de tal ferramenta está na sua praticidade e simplicidade de aplicação.

O SUS é uma derivação da escala de Likert desenvolvida em 1986, por John Brooke, para avaliar a usabilidade de uma interface. A ferramenta busca oferecer uma visão geral sobre fatores subjetivos da experiência de uso, ela não faz um diagnóstico, mas caracteriza a experiência interativa do usuário por meio de uma escala que varia de 0 a 100 pontos.

Neste trabalho, tal ferramenta foi adaptada para classificar a experiência de leitura acreditando que se algumas variáveis da composição fossem controladas seria possível a partir da variação da aparência das letras mensurar o nível de satisfação do usuário em relação a elas.

\section{Materiais e métodos}

O estudo faz uma abordagem quantitativa utilizando uma adaptação do questionário SUS para verificar o nível de satisfação entre duas fontes tipográficas, uma com serifa e outra sem.

A tarefa realizada pelos sujeitos da pesquisa foi a leitura de um texto padronizado 
Soares, J. M. R. | Protocolo SUS como ferramenta de verificação do nível de satisfação na interação com tipos com serifa e sem serifa aplicados em mídias digitais e impressas em atividade de leitura.

desenvolvido para estudos que envolvem leitura. $O$ texto foi diagramado de forma que as composições ficassem o mais próximo possível em termos tipográficos e apresentados em dois dispositivos, sendo um iPad e um protótipo em madeira similar ao iPad, neste a composição foi impressa em papel e colada no protótipo.

O procedimento realizado para a coleta de dados deste experimento solicitou que os sujeitos lessem o texto e posteriormente eles respondiam o protocolo SUS. A sequência de leitura foi determinada randomicamente estabelecida por meio do site http://www.random.org/.

Os materiais e procedimentos utilizados neste trabalho são discriminados a seguir.

\section{Amostragem}

Como o estudo envolve seres humanos no levantamento de dados, os aspectos éticos foram respeitados e a aprovação pode ser verificada por meio do protocolo CEP-FAACAprovação 580.690.

A metodologia de amostragem utilizada foi não probabilística de conveniência voluntária (Leville e Dionne, 1999), sendo composta por estudantes brasileiros, universitários, com idade entre 18 e 24 anos. Para seleção dos indivíduos participantes foi aplicada a teoria da relevância significativa (Leville e Dionne, 1999) estabelecida pelo fato de serem alunos universitários, leitores críticos (Coelho, 2000).

O tamanho da amostra foi determinado por meio da teoria de inferência estatística, que afirma ser possível determinar o tamanho da amostra com base em estudos anteriores, sendo determinada uma amostra de 84 sujeitos ( $48 \%$ do gênero masculino) divididos igualmente entre os dispositivos de leitura. Todos os participantes declararam ter acuidade visual normal, com ou sem auxílio de lentes corretivas. A coleta foi realizada em ambiente educacional, ambiente real e comum dos sujeitos da pesquisa. (Soleimani \& Mohammadi, 2012).

\section{Dispositivos de leitura}

Neste estudo foram utilizados dois dispositivos para que os sujeitos fizessem a tarefa de leitura, sendo eles: um iPad Retina (resolução 264ppi) de 9,7 polegadas, modelo da Apple MD515BR; e dois protótipos feitos de painéis de fibras de densidade média (MDF) com textos impressos em papel adesivo acrílico aquoso, da marca Pimaco-Bic (código 6285), e colados nos protótipos para expor o texto para leitura. Os dispositivos utilizados na tarefa de leitura do experimento são apresentados na figura 1.

Figura 1: Dispositivos utilizados no experimento para a realização da tarefa de leitura, na esquerda da foto é apresentado o dispositivo digital.

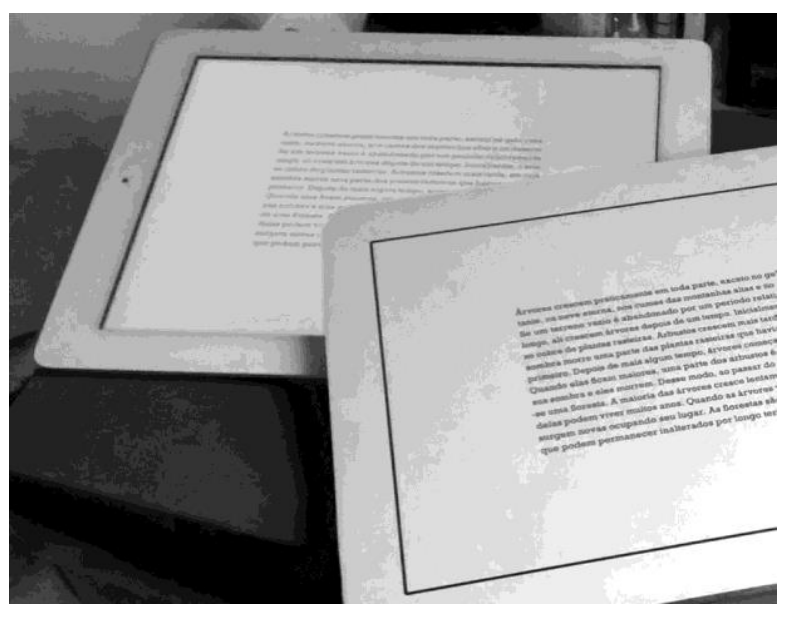


Soares, J. M. R. | Protocolo SUS como ferramenta de verificação do nível de satisfação na interação com tipos com serifa e sem serifa aplicados em mídias digitais e impressas em atividade de leitura.

\section{Diretrizes tipográficas}

O texto descrito anteriormente foi preparado em duas versões, sendo uma com um tipo com serifa (Garamond Premier Pro Regular) e outra com um tipo sem serifa (Franklin Gothic Std Book). As duas versões atender as seguintes diretrizes:

- $\quad$ Altura-x não inferior a 1,4 mm (ângulo visual (VA) 0,20ํ); (Legge e Bigelow, 2011)

- Média de caracteres por linha igual ou próxima a 66 caracteres; (Tinker, 1963 e Bringhurst, 2005)

- Espaçamento entre as linhas de 1 a 4 pontos do corpo da fonte e largura da linha entre 60 e 140 mm; (Tinker, 1963)

- Padronização dos movimentos oculares (verticais e horizontais) de leitura por meio da sistematização do número de palavras por linha e número de linhas da composição tipográfica do texto; (Dehaene, 2012)

- Equilíbrio óptico do tamanho das letras das tipografias utilizadas nas composições. (Legge e Bigelow, 2011 e Burt et al., 1955 (apud Lund, 1999))

\section{Tipos de letra do estudo}

Para este estudo foi definido o uso de dois tipos de letras: a Garamond Premier Pro regular (GP) para representar o estilo clássico dos tipos com serifa e a ITC Franklin Gothic Std Book (FG) para representar o estilo de letra sem serifa.

A escolha de tais fontes tipográficas se deu por relevância na história da tipografia, a primeira criada pelo francês Claude Garamond em 1530 e já serviu, e ainda serve, de inspiração para aqueles que buscam criar uma tipografia que reúne formas refinadas e legíveis.

A segunda, foi criada no início de 1900 pelo norte-americano Morris Fuller Benton, seus caracteres são referências da força e legibilidade dos tipos de letra sem serifa.

Figura 2: Exemplo das fontes tipográficas escolhidas para compor o texto da tarefa de leitura. Acima a fonte ITC Franklin Gothic Std Book (FG) e abaixo a fonte Garamond Premier Pro regular (GP).

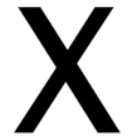

FRANKLIN GOTHIC FS BOOK (1996-2012) $10 \mathrm{pt}(1,738 \mathrm{~mm})$ Entrelinha 14p
Árvores crescem praticamente em toda parte, exceto no gelo cons tante, na neve eterna, nos cumes das montanhas altas e no deserto. Se um terreno vazio é abandonado por um período relativamente longo, ali crescem árvores depois de um tempo. Inicialmente, o solo se cobre de plantas rasteiras...

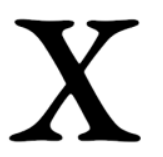

ADOBE GARAMOND PREMIER PRO (2005-2007) $12 \mathrm{pt}(1,698 \mathrm{~mm})$
Entrelinha $15 \mathrm{pt}$
Árvores crescem praticamente em toda parte, exceto no gelo constante, na neve eterna, nos cumes das montanhas altas e no deserto. Se um terreno vazio é abandonado por um período relativamente longo, ali crescem árvores depois de um tempo. Inicialmente, o solo se cobre de plantas rasteiras...

Ambos tipos de letras escolhidos são reconhecidos como criações de sucesso na história do design gráfico. As composições tipográficas foram feitas em um software de editoração eletrônica (InDesign v11.2). O ajuste realizado opticamente deixou a altura-x dos tipos utilizados no experimento com medidas acima da medida da leitura crítica, ou seja, superior a 1,4 mm ou em ângulo visual (VA) maior que 20으. VA. (Legge e Bigelow, 2011). A tabela 1 apresenta a configuração utilizada nos tipos de letras na composição do texto para a tarefa de leitura. 
Soares, J. M. R. | Protocolo SUS como ferramenta de verificação do nível de satisfação na interação com tipos com serifa e sem serifa aplicados em mídias digitais e impressas em atividade de leitura.

Tabela 1: Configuração utilizada nos tipos de letras na tarefa de leitura.

\begin{tabular}{l|l|l|l|l}
$\begin{array}{l}\text { Fonte } \\
\text { tipográfica }\end{array}$ & $\begin{array}{l}\text { Corpo/ } \\
\text { entrelinha }\end{array}$ & Altura-X & $\begin{array}{l}\text { Ângulo } \\
\text { Visual }\end{array}$ & $\begin{array}{l}\text { Uso de } \\
\text { Serifa }\end{array}$ \\
\hline Garamond (GP) & $12 / 15 \mathrm{pt}$ & $1,65 \mathrm{~mm}$ & $0.24^{\circ}$ & Sim \\
\hline Franklin Gothic (FG) & $10 / 14 \mathrm{pt}$ & $1,77 \mathrm{~mm}$ & $0.25^{\circ}$ & Não \\
\hline
\end{tabular}

\section{Padronização de texto}

Para a tarefa de leitura foi adotado um texto padronizado para estudos multicêntricos internacionais que envolvem leitura. O texto é composto por 831 caracteres organizados em 132 palavras e apresentam versões equivalentes em quatro idiomas (alemão, finlandês, francês e inglês). (Messias et. al, 2008).

O conteúdo do texto trata do tema ecologia e a linguagem é adequada para as habilidades intelectuais de crianças de 12 anos de idade que equivale a alunos do sétimo ano do ensino fundamental do sistema de ensino brasileiro vigente, portanto, sem complexidade para estudantes de curso universitário.

Todas as composições apresentam 13 linhas e 67 caracteres por linha, em média, organizados em uma média de 11 palavras por linha. A figura 3 apresenta uma amostra da composição final do texto nos dois dispositivos de leitura.

Figura 3: Exemplo da composição utilizada para tarefa de leitura, a direita foto do texto na tela do dispositivo digital.

Árvores crescem praticamente em toda parte, exceto no gelo cons-
tante, na neve eterna, nos cumıss das montanhas altas e no deserto.
Se um terreno vazio é abandorıado por um período relativamente
longo, ali crescem árvores depcis de um tempo. Inicialmente, o solo
se cobre de plantas rasteiras. A rbustos crescem mais tarde, em cuja
sombra morre uma parte das plantas rasteiras que haviam surgido
primeiro. Depois de mais algunı tempo, árvores começam a crescer.
Quando elas ficam maiores, unıa parte dos arbustos é atingida por
sua sombra e eles morrem. Des se modo, ao passar do tempo, forma-
-se uma floresta. A maioria das d́rvores cresce lentamente, algumas
delas podem viver muitos anos. Quando as árvores velhas morrem,
surgem novas ocupando seu lv.gar. As florestas são espaços vitais
que podem permanecer inalte::ados por longo tempo.

\section{Adaptação do protocolo SUS}

O protocolo SUS consiste em um questionário de fatores subjetivos com 10 enunciados, dos quais cinco são afirmações positivas e cinco negativas para mensurar a experiência interativa do usuário. As questões foram adaptadas para o contexto tipográfico para que os participantes do estudo, após a leitura do texto, pudessem indicar o que mais se aproximou da sua opinião. A figura 4 apresenta o protocolo SUS adaptado para a coleta deste estudo. 
Soares, J. M. R. | Protocolo SUS como ferramenta de verificação do nível de satisfação na interação com tipos com serifa e sem serifa aplicados em mídias digitais e impressas em atividade de leitura.

Figura 4: Imagem do protocolo SUS adaptado para o teste de satisfação tipográfica.

\begin{tabular}{|c|c|c|c|}
\hline $\begin{array}{l}\text { 1. Eu gostaria que o aspecto do texto } \\
\text { fosse usado com mais frequência } \\
\text { nos materiais de leitura. }\end{array}$ & $\begin{array}{l}\text { Discordo } \\
\text { Totalmente }\end{array}$ & $\square \square \square \square \square$ & $\begin{array}{l}\text { Concordo } \\
\text { Totalmente }\end{array}$ \\
\hline $\begin{array}{l}\text { 2. Achei o aspecto do texto } \\
\text { desnecessariamente enfeitado. }\end{array}$ & $\begin{array}{l}\text { Discordo } \\
\text { Totalmente }\end{array}$ & $\square \square \square \square \square$ & $\begin{array}{l}\text { Concordo } \\
\text { Totalmente }\end{array}$ \\
\hline $\begin{array}{l}3.0 \text { aspecto do texto sugere uma } \\
\text { leitura muito mais fácil. }\end{array}$ & $\begin{array}{l}\text { Discordo } \\
\text { Totalmente }\end{array}$ & $\square \square \square \square \square$ & $\begin{array}{l}\text { Concordo } \\
\text { Totalmente }\end{array}$ \\
\hline $\begin{array}{l}\text { 4. Achei que o aspecto do texto } \\
\text { complicou um pouco a minha leitura. }\end{array}$ & $\begin{array}{l}\text { Discordo } \\
\text { Totalmente }\end{array}$ & $\square \square \square \square \square$ & $\begin{array}{l}\text { Concordo } \\
\text { Totalmente }\end{array}$ \\
\hline 5. Achei o aspecto do texto bem equilibrado. & $\begin{array}{l}\text { Discordo } \\
\text { Totalmente }\end{array}$ & $\square \square \square \square \square$ & $\begin{array}{l}\text { Concordo } \\
\text { Totalmente }\end{array}$ \\
\hline $\begin{array}{l}\text { 6. Eu achei o aspecto do texto } \\
\text { pouco agradável. }\end{array}$ & $\begin{array}{l}\text { Discordo } \\
\text { Totalmente }\end{array}$ & $\square \square \square \square \square$ & $\begin{array}{l}\text { Concordo } \\
\text { Totalmente }\end{array}$ \\
\hline $\begin{array}{l}\text { 7. Imagino que o aspecto do texto } \\
\text { deixa a leitura bem mais rápida e } \\
\text { simples para a maioria das pessoas. }\end{array}$ & $\begin{array}{l}\text { Discordo } \\
\text { Totalmente }\end{array}$ & $\square \square \square \square \square$ & $\begin{array}{l}\text { Concordo } \\
\text { Totalmente }\end{array}$ \\
\hline $\begin{array}{l}8.0 \text { aspecto do texto é pouco } \\
\text { convidativo à leitura. }\end{array}$ & $\begin{array}{l}\text { Discordo } \\
\text { Totalmente }\end{array}$ & $\square \square \square \square \square$ & $\begin{array}{l}\text { Concordo } \\
\text { Totalmente }\end{array}$ \\
\hline $\begin{array}{l}9.0 \text { aspecto do texto me deu } \\
\text { segurança para a leitura. }\end{array}$ & $\begin{array}{l}\text { Discordo } \\
\text { Totalmente }\end{array}$ & $\square \square \square \square \square$ & $\begin{array}{l}\text { Concordo } \\
\text { Totalmente }\end{array}$ \\
\hline $\begin{array}{l}\text { 10. Demorei mais tempo na } \\
\text { leitura do que imaginei. }\end{array}$ & $\begin{array}{l}\text { Discordo } \\
\text { Totalmente }\end{array}$ & $\square \square \square \square \square$ & $\begin{array}{l}\text { Concordo } \\
\text { Totalmente }\end{array}$ \\
\hline
\end{tabular}

\section{Procedimentos de coleta}

A coleta de dados iniciou-se com a distribuição da amostra em quatro grupos, sendo dois deles para cada um dos dois tipos de letras do estudo, um representante dos tipos com serifa e outro sem serifa. E para cada tipo de letra foram utilizados dois suportes para a tarefa de leitura, um digital e outro analógico com o texto impresso em papel, posteriormente foram adotadas as seguintes etapas:

1. Abordagem dos usuários, explicação do estudo e convite para que eles participassem da pesquisa em questão;

2. Esclarecimento dos objetivos e apresentação do termo de consentimento livre e esclarecido;

3. Leitura do texto padronizado conforme lista randômica da sequência predefinida;

4. Aplicação do protocolo SUS;

5. Tabulação e processamento estatístico de dados.

\section{Resultados}

Após obtenção das medidas de tendência central, os dados foram analisados no programa

Statistica, software utilizado para realizar o teste de normalidade (Histograma/ShapiroWilk's/Wilcoxon) das amostras coletadas.

As figuras 5 e 6 ilustram a comparação e detalham os resultados obtidos no estudo. Nela

Anais do 9 Congresso Internacional de Design da Informação | CIDI 2019 
Soares, J. M. R. | Protocolo SUS como ferramenta de verificação do nível de satisfação na interação com tipos com serifa e sem serifa aplicados em mídias digitais e impressas em atividade de leitura.

é possível verificar por meio do teste T. Wilcoxon que não há diferença significativa $(p>$ $0,05)$ entre os valores obtidos na escala de satisfação do protocolo SUS para cada um dos tipos de letras pesquisados, sendo um tipo de letra sem serifa representado pela fonte tipográfica ITC Franklin gothic Std Book (FG) e outro com serifa expresso pela Garamond Premier Pro Regular (GP).

Figura 5: Gráfico apresentando um comparativo entre o resultado dos níveis de satisfação dos tipos de letras analisados em dispositivos de leitura diferentes (ITC Franklin gothic Std Book" (FG) and the "Garamond Premier Pro Regular" (GP)).

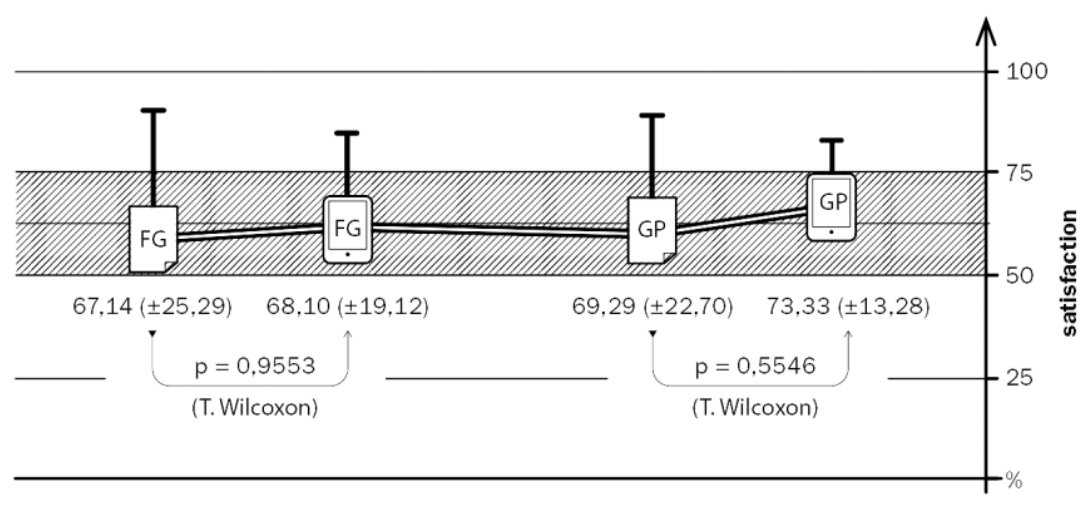

Figura 6: Gráfico apresentando um comparativo entre o resultado dos níveis de satisfação dos tipos de letras analisados (ITC Franklin gothic Std Book" (FG) and the "Garamond Premier Pro Regular" (GP)).

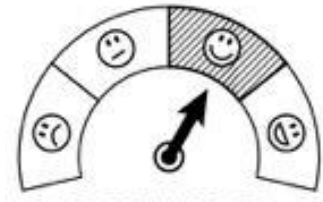

$67,62 \%( \pm 22,15)$

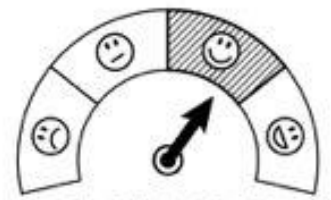

$71,31 \%( \pm 18,48)$
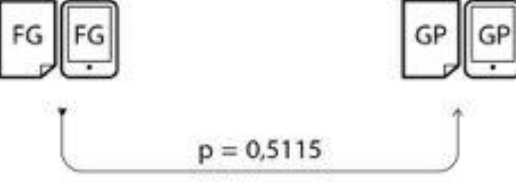

(T. Wilcoxon)

\section{Conclusões}

Por meio do experimento realizado, comparando dois tipos de letras de estética diferentes, mas tendo sua altura- $x$ e entrelinha equivalentes opticamente, número de palavras por linha e número de linhas do texto, a composição tipográfica não interferiu de forma significativa na satisfação do leitor na tarefa da leitura.

Em tal contexto, pode-se deduzir que o arranjo tipográfico pode favorecer ou comprometer o uso de um estilo de letra. Faz-se necessário mais estudos para uma conclusão definitiva, mas, a princípio pode-se dizer que a maneira como um tipo de letra é utilizada se sobrepõe à escolha dela, desde que ela seja comum ao leitor em pauta.

Com base nos resultados deste estudo é possível concluir que o uso do protocolo SUS como ferramenta de avaliação para mensurar o nível de satisfação da tipografia é admissível, uma vez que ratifica que a escolha do tipo de letra, quando esta for comum, pouca influência terá sobre a tarefa de leitura. A metodologia e protocolo utilizado ratificam resultados já demonstrados por outros autores, como Beier, Sand e Starrfelt (2017); Soares 
Soares, J. M. R. | Protocolo SUS como ferramenta de verificação do nível de satisfação na interação com tipos com serifa e sem serifa aplicados em mídias digitais e impressas em atividade de leitura.

(2016); Soleimani \& Mohammadi (2012) e Arditi \& Cho (2005) reforçando o preceito da "invariância perceptiva" na publicação Neurônios da Leitura exposto por Dehaene (2012).

\section{Referências}

Beier, S., SAND, K., \& STARRFELT, R. (2017). Legibility Implications of Embellished Display Typefaces. Visible Language, 51(1), 112-133. Acesso em: 04 jul. 2019.

Beier, Sofie (2009). Typeface legibility: towards defining familiarity (Doctoral dissertation). London: Royal College of Art. Disponível em: <http://dolp.cc/3IF2>. Acesso em: 15 jan. 2016.

Bringhurst, Robert (2005). Elementos do estilo tipográfico. Tradução André Stolarski. São PauloEditora Cosac Naify, p423.

Brooke, John (1996). SUS-A quick and dirty usability scale. Usability evaluation in industry, v.189, n.194, p4-7. Disponível em: <http://dolp.cc/qZMH>. Acesso em: 15 jan. 2016.

Chung, Susana T.L (2007). Learning to identify crowded letters: does it improve reading speed?. Vision research, v.47, n.25, p3150-3159. Disponível em: <http://dolp.cc/YZzN>. Acesso em: 15 jan. 2016.

Dehaene, Stanislas (2012). Os neurônios da leitura. Trad. de L. Scliar-Cabral. Porto Alegre: Penso, 2012.

Geske, Joel (1996). Legibility of Sans Serif Type for use as Body Copy in Computer Mediated Communication. Disponível em: <http://dolp.cc/RD7w>. Acesso em: 15 jan. 2016.

Lund, O. (1999). Knowledge construction in typography: the case of legibility research and the legibility of sans serif typefaces (Doctoral dissertation). Disponível em: <http://dolp.cc/M2HB>. Acesso em: 15 jan. 2016.

LEGGE, Gordon E.; Bigelow, Charles A (2011). Does print size matter for reading? A review of findings from vision science and typography. Journal of Vision, 11(5), 8. Disponível em: <http://dolp.cc/WZ5c>. Acesso em: 15 jan. 2016.

Leville, C. \& DIONNE, J. (1999). A construção do saber. Porto Alegre: Editora UFMG.

Messias, André; Cruz, Antonio A. V. e; Schallenmüller, Sonia J.; Trauzettel-Klosinski, Susanne (2008). Textos padronizados em português (BR) para medida da velocidade de leitura-comparação com quatro idiomas europeus. Arq. Bras. Oftalmol., v.71, n.4, p553558. Disponível em: <http://dolp.cc/1njH>. Acesso em: 15 jan. 2016.

Soares, João Marcelo Ribeiro. Design gráfico ergonômico: método para verificação de níveis de usabilidade de fontes tipográficas para texto em suportes impressos e digitais. 2016. Disponível em: <http://hdl.handle.net/11449/138159>. Acesso em: 04 jul. 2019.

Soleimani, H. \& Mohammadi, E. (2012). The Effect of Text Typographical Features on Legibility, Comprehension, and Retrieval of EFL Learners. English Language Teaching, 5(8), p207. Disponível em: <http://dolp.cc/ZVR0>. Acesso em: 15 jan. 2016.

Tinker, Miles A. (1963). Legibility of print. Ames: lowa State University Press, 1963.

\section{Sobre o autor}

João Marcelo Ribeiro Soares, Doutor em Design pela Unesp-SP, Professor no curso de Design da Universidade do Sagrado Coração (USC) de Bauru-SP, Brasil,

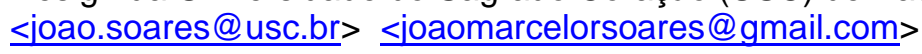

